\title{
बु \\ Magnetic properties of vortex states in spherical superconductors
}

\author{
Ben Xu, ${ }^{1}$ M. V. Milošević,,${ }^{1,2}$ and F. M. Peeters ${ }^{1, *}$ \\ ${ }^{1}$ Departement Fysica, Universiteit Antwerpen, Groenenborgerlaan 171, B-2020 Antwerpen, Belgium \\ ${ }^{2}$ Department of Physics, University of Bath, Claverton Down, BA2 7AY Bath, United Kingdom \\ (Received 16 January 2008; revised manuscript received 1 March 2008; published 7 April 2008)
}

\begin{abstract}
Using a fully three-dimensional numerical treatment of the Ginzburg-Landau (GL) theory, we study superconducting and magnetic properties of submicron superconducting spheres. Systematic analysis is performed for different radii of the sphere and different constituent materials, i.e., with different GL parameter $\kappa$. The distribution of the magnetic field in and around the sample is calculated, and its repercussions on magnetometry measurements are discussed. Furthermore, we demonstrate a unique feature in the magnetization curves that can distinguish multi- from giant-vortex states.
\end{abstract}

DOI: 10.1103/PhysRevB.77.144509

PACS number(s): 74.20.De, 74.25.Dw, 74.78.Na, 74.25.Ha

\section{INTRODUCTION}

Recent advances in nanofabrication techniques will enable fast preparation and experimental studies of truly threedimensional (3D) mesoscopic superconductors. ${ }^{1-3}$ It is already known that quantum confinement in thin mesoscopic superconductors, imposed by the sample geometry, has important consequences on both vortex matter and critical properties of the sample. In such essentially two-dimensional (2D) superconductors with sizes comparable to the penetration depth $\lambda$ and coherence length $\xi$, numerous works have been done over the past decades, theoretically by Schweigert and Peeters, ${ }^{4,5}$ Palacios ${ }^{6,7}$ solving nonlinear GinzburgLandau equations, and Buzdin and Brision ${ }^{8}$ using the image method within the London approximation and also experimentally by Moshchalkov and co-workers, ${ }^{9,10}$ Geim et $a l .,{ }^{11,12}$ and Kanda and co-workers. ${ }^{13,14}$ The effect of the geometry of the sample on superconductivity has also been thoroughly investigated. ${ }^{15-21}$ However, sample geometry was a two-dimensional quantity in the latter studies, as the superconducting order parameter was always uniform along the $z$ axis. This is obviously not the case in three-dimensional samples (e.g., sphere), where the slanted angle of the sample sides with respect to the applied magnetic field plays a significant role. Therefore, those earlier theories based on the 2D London approximation become incapable, and until now, no corresponding theory approximation is proposed for 3D cases. So the Ginzburg-Landau study of the interplay of the applied magnetic field and 3D distribution of the Cooper-pair density is the first important objective of this paper.

In previously studied thin mesoscopic samples, ${ }^{5}$ two fundamental kinds of vortex states were theoretically predicted: (i) the multivortex state with a spatial arrangement of single vortices (each carrying one flux quantum) and (ii) axially symmetric giant-vortex state, where several vortices coalesce into a single core, containing multiple flux quanta. The existence of those vortex states in superconducting disks was recently verified in an experiment using the multiple-smalltunnel-junction method. ${ }^{13}$ However, the definition of giantand multivortex states becomes more complicated if the problem is extended to the third dimension, especially in more complicated sample geometries, where vortices are exposed to a complicated 3D interaction with the sample boundary. In previous work, ${ }^{22}$ a breakup of the vortex structure in a 3D mesoscopic wire with a constriction was predicted. A giant vortex nucleated in the widest part of the wire and split into a smaller and/or individual vortices near the constriction. Here, we study the vortex states in spherical mesoscopic samples (with different radii), and elsewhere for different material properties (i.e., Ginzburg-Landau parameter $\kappa$ ), which fine tune the vortex-vortex interaction. ${ }^{23}$

The understanding of the effects of the true three dimensionality of the studied system is not only important for theoretical purposes but also for experiment as well. In transport measurements, it now becomes crucial where to attach current and/or voltage leads to the sample. Similarly, in magnetometry, the exact position of the placed Hall probe above the sample is much more important than for thin 2D samples. In this work, we partly address the latter issue and calculate the stray field around the sample (thus in 3D), the magnetic moment of the sample as a whole, as well as the response of the Hall bar as a function of its position and size.

Our present work is not only a continuation but also a considerable extension of the existing studies of spherical superconductors. In Ref. 24, vortex matter in a superconducting sphere was studied using an approach based on the linear Ginzburg-Landau (GL) theory. However, this approach is limited to extreme type-II superconductors, and the local magnetic response of the sample was completely neglected. Vortex patterns in superconducting shells were studied in Ref. 25, where no demagnetization effects were taken into account. Reference 26 dealt with the effects of the chosen boundary condition on the vortex patterns in mesoscopic thick disks and spheres, but still remaining in the $\kappa \rightarrow \infty$ limit. Within the same limit, finite size mesoscopic cylinders and asymmetric spheres were studied in a tilted magnetic field. ${ }^{27}$ In the present work, we study superconducting spheres with magnetic screening fully accounted for (for different $\kappa$ ) within the Ginzburg-Landau formalism solved on a 3D grid. Therefore, both the superconducting order parameter and the local magnetic field are calculated selfconsistently in 3D space.

The paper is organized as follows. In Sec. II, we introduce the theoretical model. In Sec. III, we present the phase diagram for all vortex states as a function of the sample size and applied magnetic field. The importance of the chosen super- 
conducting material (with different Ginzburg-Landau parameter $\kappa$ ) for the vortex behavior is studied in Sec. IV. The simulations of the magnetic response of the sample are shown in Sec. V, where the assumed finite size of the Hall bar in magnetometry measurements is taken into account. In Sec. VI, we summarize our findings.

\section{THEORETICAL FORMALISM}

In what follows, we consider superconducting spheres characterized by their radius $R$, and GL parameter $\kappa=\lambda / \xi$, where $\xi$ and $\lambda$ are coherence length and penetration depth, respectively. We assume all considered samples to be immersed in an insulating medium and exposed to a uniform magnetic field $\vec{H}=(0,0, H)$. To numerically treat this system, we follow the numerical approach of Schweigert and Peeters. ${ }^{4}$ First, using dimensionless variables and the London gauge $\operatorname{div} \vec{A}=0$ for the vector potential $\vec{A}$, we rewrite the system of two coupled GL equations in the following form:

$$
\begin{gathered}
\left(-i \vec{\nabla}_{3 D}-\vec{A}\right)^{2} \Psi=\Psi\left(1-|\Psi|^{2}\right), \\
-\kappa^{2} \Delta_{3 D} \vec{A}=\vec{j}_{3 D},
\end{gathered}
$$

where

$$
\vec{j}_{3 D}=\frac{1}{2 i}\left(\Psi^{*} \vec{\nabla}_{3 D} \Psi-\Psi \vec{\nabla}_{3 D}^{*} \Psi\right)-|\Psi|^{2} \vec{A}
$$

is the density of the superconducting current induced by the sample in response to the applied field. Here, the distance is measured in units of the coherence length $\xi$, the vector potential in $c \hbar / 2 e \xi^{2}=\kappa \sqrt{2} H_{c}$, and order parameter is scaled to its value in the absence of the magnetic field. Equations (1) and (2) are then discretized on a uniform cubic grid using the link variable approach, ${ }^{28}$ in Cartesian coordinates with typically five grid points per $\xi$, and solved in a finite-difference scheme. Equation (2) is solved using three-dimensional fast Fourier transform in a method similar to the known procedures for solving the Poisson equation (see, e.g., Ref. 29).

In our calculations, the superconducting order parameter satisfies the Neumann boundary condition,

$$
\left.\left(-i \vec{\nabla}_{3 D}-\vec{A}\right) \Psi\right|_{\vec{\rho}=\vec{R}}=0,
$$

while the external applied vector potential has the symmetric form,

$$
\vec{A}_{x}=-\frac{1}{2} H y \vec{e}_{x}, \quad \vec{A}_{y}=\frac{1}{2} H x \vec{e}_{y}, \quad \vec{A}_{z}=0 .
$$

Additional constriction is that the resulting (total) vector potential must decay to zero $(\vec{A}=0)$ far away from the superconductor.

To find the different vortex configurations, which include all stable states (thus the lowest energy ground state and the higher energy metastable states), we search for the selfconsistent solutions of Eqs. (1) and (2). If we simulate a field-cooled experimental situation, we initiate the calculation from randomly generated initial conditions (for a zerofield-cooled situation, we start from $\Psi \approx 1$ in the whole

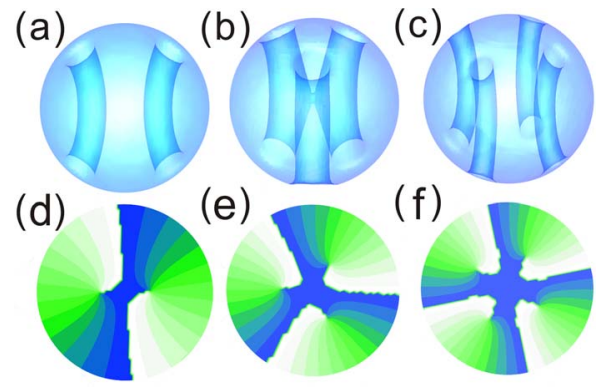

FIG. 1. (Color online) Three-dimensional 10\% isoplots of the Cooper-pair density in the sphere with radius $R=4 \xi$ for vorticities (a) $L=2$ at applied magnetic field $H=0.61 H_{c 2}$, (b) $L=3$ at $H$ $=0.79 H_{c 2}$, and (c) $L=4$ at $H=1.14 H_{c 2}$. (d) $-(\mathrm{f})$ show the corresponding phase contourplots in the equatorial plane of the sample.

sample). Then, we slowly change the applied magnetic field and recalculate each time the superconducting state. In such a way, we are able to trace back and forth all found vortex states in the whole region of their stability. For each vortex configuration, found at different applied fields, we calculate the Gibbs free energy (in units of $F_{0}=H_{c}^{2} / 4 \pi$ ) as

$$
F=V^{-1} \int_{V}\left[2\left(\vec{A}-\vec{A}_{0}\right) \cdot \vec{j}_{3 D}-|\Psi|^{4}\right] d \vec{r},
$$

where integration is performed over the sample volume $V$ and $\vec{A}_{0}$ is the vector potential of the initially applied magnetic field.

To characterize the diamagnetic property of our superconducting sample, we calculate its magnetization in applied field. Differently from the thermodynamic expression $M$ $=\partial F / \partial H$, we define sample magnetization as the expelled magnetic field from the sample, i.e., as

$$
\vec{M}=\frac{\langle\vec{h}\rangle-\vec{H}}{4 \pi},
$$

where $\vec{H}$ is the applied magnetic field and $\vec{h}=\operatorname{rot} \vec{A}$ is the resulting (total) magnetic field. Here, \langle\rangle denotes averaging over the particular area (which could be the sample as a whole, or just a surface area of a magnetic detector, e.g., Hall probe, at a particular location above the sample).

\section{VORTEX STATES IN A MESOSCOPIC SUPERCONDUCTING SPHERE}

In this section, we will discuss fundamental properties of the vortex configurations in spherical superconductors. Knowing that the density of the superconducting Cooper pair drops to zero inside the vortex core, we show the 3D isoplots of the low Cooper-pair density inside the sample to best illustrate the vortex configuration. Such plots are shown in Fig. 1, each with a corresponding plot of the phase of the order parameter (in the equatorial plane), for states with vorticities [(a) and (d) $L=2$, [(b) and (e) $L=3$, and [(c) and (f)] $L=4$. According to the definition of vorticity in Ref. 4, the total number of vortex lines trapped in the sample, which can be deduced from the number of $0 \rightarrow 2 \pi$ phase, changes along 


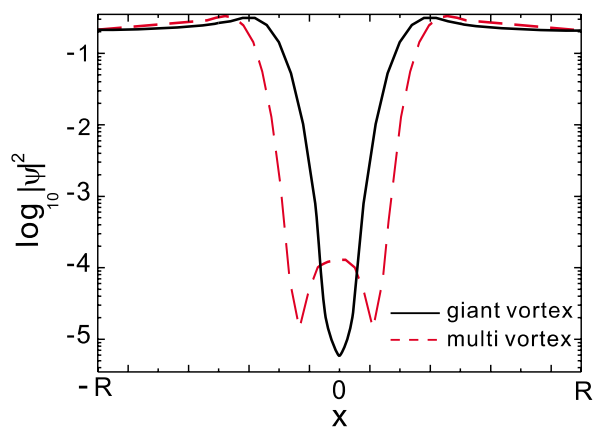

FIG. 2. (Color online) Illustration of the giant-vortex and the multivortex states for $L=2$, at applied magnetic fields $H=0.81 H_{c 2}$ and $0.76 H_{c 2}$, respectively, close to the numerical ambiguity. The Cooper-pair density is plotted along the sample diameter, where found minima are taken as singularities of the order parameter.

the sample rim in Figs. 1(d)-1(f). Contrary to the straight vortex lines in the case of superconducting cylinder and/or disk, in our sample, vortices are compressed together in the equatorial plane of the sample, due to the strong screening (Meissner) currents running along the perimeter; at the same time, vortices remain perpendicular to the surface of the sample edge at the point of entry and/or exit, which results in their strong bowing along the sample (see Fig. 1).

Even though compressed in the center of the sample, individual vortices are still clearly separated in both $|\Psi|^{2}$ isoplots and phase contourplots in Fig. 1. We refer to such vortex state as a multivortex (MV) state in the remainder of the paper. However, this nomination may become ambiguous for overlapping vortex lines, which can be expected at higher fields, and a clear numerical threshold must be established to differentiate the MV state from a giant-vortex (GV) state. In Fig. 2, we illustrate the used criterion in our calculation, where the Cooper-pair density is plotted across the center of the sample. When a maximal value between found minima of the Cooper-pair density exceeds $10^{-5}$, we regard the found state as a collection of singularities, i.e., a multivortex state. Otherwise, the numerical precision may be questioned, and we assume a single singularity, characteristic of a giant vortex.

The main principle of quantum confinement of vortices in mesoscopic superconductors follows from the sample size being comparable to the size of the vortex core. Therefore, we briefly address the influence of our sphere's size on the vortex configurations. In Fig. 3, the free energy curves are given as a function of the applied magnetic field for a superconducting sphere with radii (a) $R=3 \xi$ and (b) $R=4 \xi$. Different energy curves with increasing field correspond to states with gradually incremented vorticity, starting from the vortex-free, Meissner phase $(L=0)$. As can be seen in Fig. 3, more vortices can be captured in the larger sample before superconductivity is destroyed. Maximal vorticity is $L_{\max }$ $=9$ for $R=4 \xi$, compared to $L_{\max }=4$ for $R=3 \xi$. The screening of the magnetic field is much more effective in the smaller samples, where the penetration field for vortices is significantly larger [in Fig. 3(a), $H_{p}=0.902 H_{c 2}$, compared to $H_{p}$ $=0.45 H_{c 2}$ in Fig. 3(b)]. Namely, a larger superconducting sphere proportionally expels more magnetic flux in the

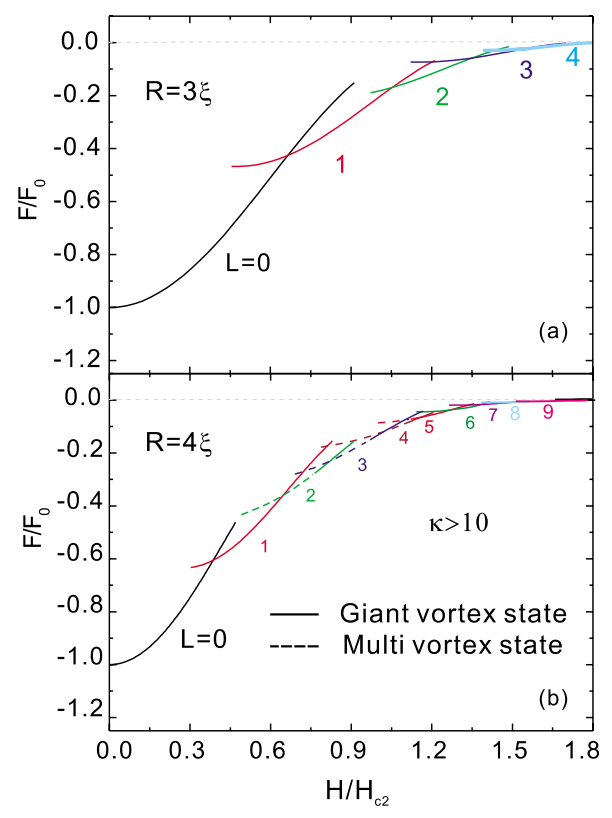

FIG. 3. (Color online) The free energy curves as a function of the applied magnetic field for spheres with radii (a) $R=3 \xi$ and (b) $R=4 \xi$. Giant-vortex states are indicated by solid curves, while multivortex states are denoted by dashed curves.

Meissner phase, which results in a higher field at the equatorial boundary of the sample for a larger sample than for the smaller ones at the same applied field. Such high magnetic field around larger samples suppresses superconductivity at the sample edge and creates weak points that facilitate the entry of vortices.

Another influence of the confinement can be observed for larger vorticity. In Fig. 3(a), penetrating flux lines are strongly influenced by the proximity of the boundary and are forced to coalesce into a giant vortex for all vorticities. For larger samples [e.g., the one in Fig. 3(b)], latter condition is relaxed, and singly-quantized vortices may remain stable in the sample. The solid lines in Fig. 3(b) represent the giantvortex state, while the dashed lines show the multivortex state. In general, one notes that multivortex states are initially formed, as vortices individually enter from the sample boundary. With increasing field, the screening currents strengthen at the equator, compressing the vortices together, which eventually results in the formation of a giant vortex. In short, MV/GV state transition is a product of the competing interactions in the system, as individual vortices repulsively interact with currents at sample edges (inward force), as well as with each other (outward force). As a comparison, the free energy curves for different vortex states are plotted for a cylinder in Fig. 4, with the same radius of $4 \xi$ and height of $5.5 \xi$ (giving the same volume as the considered sphere). The confinement from the curved boundary of the sphere is replaced by the relatively "looser" and homogenous ones from the cylinder. Thus, more vortices can be formed inside the sample, and multivortex states are preferred to the giantvortex states for the same vorticity.

To systematically study the behavior of vortices for different sizes of the superconducting sphere, we varied the 


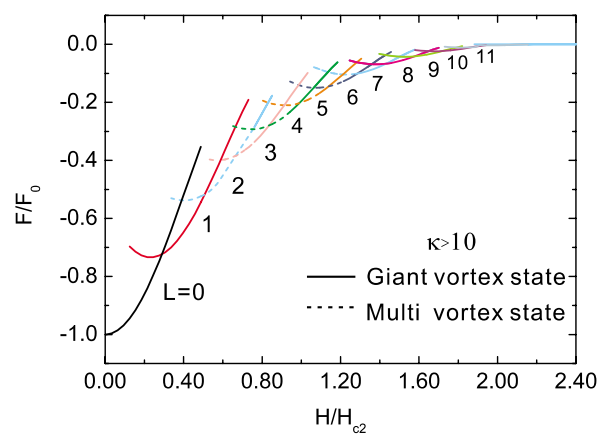

FIG. 4. (Color online) The free energy curves as a function of the applied magnetic field for a cylinder with radius $R=4 \xi$ and height $d=5.5 \xi$. Giant-vortex states are indicated by solid curves, while multivortex states are denoted by dashed curves.

radius of the sample from $1.0 \xi$ to $4.6 \xi$ and recorded the ranges of applied magnetic field in which each of the vortex states represented the ground state of the system. Results are summarized in the phase diagram of Fig. 5 for the GinzburgLandau parameter $\kappa=10$. Three main parts can be identified in Fig. 5-the Meissner phase (left bottom), normal state (i.e., destroyed superconductivity, top right), and the mixed (vortex) state (between the other two). The upper critical field of the sample is found to be $1.78 H_{c 2}$ for $R=4.5 \xi$, which is $\sim 80 \%$ larger than the critical field of bulk samples, and increases further as the sphere is made smaller [dramatic increase is found for $R<2 \xi$ (see Fig. 5)].

The vortex state region in the equilibrium vortex phase diagram is very rich, as the maximal vorticity in the sample rises to $L_{\max }=13$ for $R=4.6 \xi$. As explained earlier, larger samples favor the formation of multivortex states, as is indeed shown by the shaded area in Fig. 5. Moreover, one should note that for fixed vorticity and increasing size of the sample, we find a lower nucleation field for the MV state, but a higher transition field to a GV state (dashed lines). Found transition lines between different $L$ states seem to be mostly equidistant in the phase diagram, as they should be due to the flux quantization effects. In addition, the aforementioned

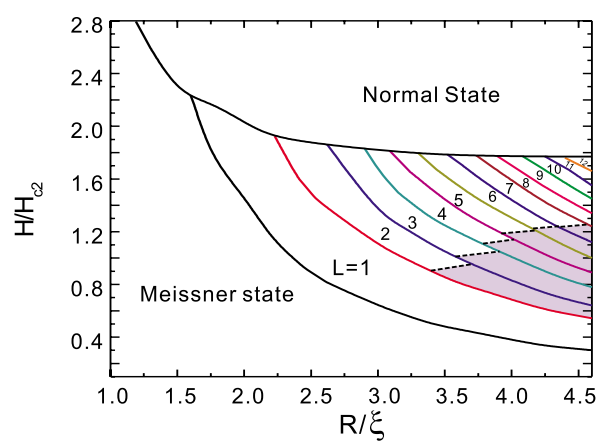

FIG. 5. (Color online) The phase diagram for the ground-state vortex configurations as a function of the applied magnetic field $H$ and the radius of the superconducting sphere (for $\kappa=10$ ). Different vortex states are separated by solid curves, while the giant- to multivortex transitions are denoted by dashed curves. Shaded areas correspond to the multivortex regions.

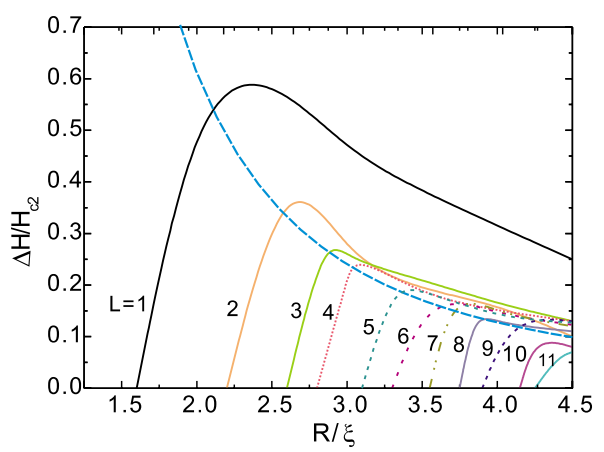

FIG. 6. (Color online) The ground-state magnetic field range $\Delta H$ for different vortex states as a function of the sample size. Hyperbolic dashed curve gives the parameters for which exactly $\Delta \Phi=\Phi_{0}$ is applied through the equatorial plane of the sample.

curves have paraboliclike behavior, suggesting their $R^{2}$ dependence remnant of the flux quantization through the equatorial plane of the sample. However, it is apparent that the $L=1$ state shows a pronounced stability in comparison with other states. To illustrate this better, we constructed Fig. 6, in which the ground-state field-stability region $\Delta H$ is given for each state as a function of the sphere radius.

The found nonmonotonic behavior of $\Delta H$ follows from initial hampering of the stability region by destruction of superconductivity. Only after the vortex state is succeeded as one of the higher vorticities can $\Delta H$ reach its full extent, which results in the corresponding maximum in all curves shown in Fig. 6. However, it is most interesting to observe the location of these maxima with respect to the dashed line in Fig. 6, which gives $\Delta H$ and $R$ needed for the addition of exactly one flux quantum to the system. While the maxima (and the follow-up points) of higher vorticity curves nicely sit on or just above the curve $\Phi_{0}=\pi c \hbar / 2 e$, the curve for the $L=1$ state significantly deviates from the expected values. The fact that larger flux is needed for the penetration of the first vortex in the mesoscopic sample is already a known fact $^{30}$ [in here considered samples $\left.\Phi_{0 \rightarrow 1}=(2.5-3.0) \Phi_{0}\right]$, but pronounced stability of the $L=1$ state with respect to applied flux $\left(\Delta \Phi=2.36 \Phi_{0}\right)$ is a unique property of spherical superconductors, caused by both symmetry and the three dimensionality of the sample. In the single-vortex state, the vortex line connects the poles of the sphere, ideally placed in the center of the sample. In such a symmetric configuration, vortex currents ideally compensate the increasing screening currents, which prolongs further flux entry. In addition, the new vortex line breaks the existing radial symmetry, and also causes either shortening or bowing of the first vortex, due to its new off-center position. All of these processes cost energy and increase the energy barrier for flux penetration. For similar reasons, further flux entry (e.g., $L=2$ to $L=3$ transition) is more energetically favorable as (i) distinct weak points are created for vortex entry (e.g., $n$ weak points between $L=n$ existing vortices on a ring) and (ii) existing vortices rearrange in a new configuration while keeping similar selfgeometry. 


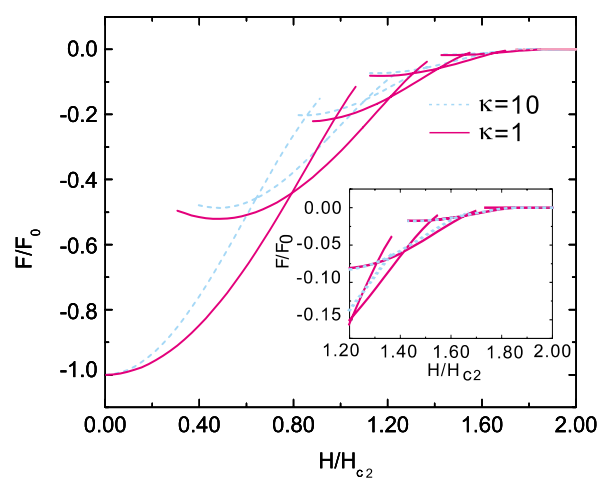

FIG. 7. (Color online) The free energy as a function of the applied magnetic field for samples with $R=3 \xi$ and $\kappa=10.0$ (dashed lines) and 1.0 (solid lines). The inset zooms in the high-field region of the diagram.

\section{VORTEX BEHAVIOR IN SPHERES OF DIFFERENT MATERIALS}

In previous section, we considered a rather extreme type-II superconducting sphere, with GL parameter $\kappa>10$ (for more information, see also Refs. 24 and 26). However, very few low $-T_{c}$ superconducting materials are known to be of such type, except, e.g., $\mathrm{NbSe}_{2}$, which is also anisotropic (in high- $T_{c}$ materials, $\kappa$ is also large, but coupling between layered structure of the cuprate planes must be taken into account $\left.^{31}\right)$. From a theoretical point of view, the issue of lower $\kappa$ is rather important, as it means that screening currents can no longer be marginalized in the data analysis, and calculation also becomes much more numerically demanding [i.e., the influence of Eq. (2) in our formalism rises]. When considering vortex matter, $\kappa$ is one of the crucial sample properties as it governs the behavior of the vortex-vortex interaction. Therefore, contrary to thin samples which in most cases exhibit extreme type-II behavior (where effective GL parameter is defined as $\kappa^{*}=\kappa^{2} / d, d$ being the thickness), properties of 3D superconducting samples directly depend on the characteristic lengths of the constituent material. For comparison, in this section, we consider samples with $\kappa$ $<10$, with some emphasis on the $\kappa \approx 1$ case, thus, e.g., niobium spheres.

To begin with, we compare two samples with radius $R$ $=3 \xi$ and $\kappa$ equal to 10 and 1 , respectively. In Fig. 7, the free energy curves for those samples are plotted versus the applied magnetic field. One can see that transitions between successive vortex states move to higher fields when $\kappa$ is decreased, and the superconducting and/or normal state transition occurs at somewhat higher applied field. We carefully studied these features by taking finer steps when changing $\kappa$ between values of 0.71 and 10 . Results are summarized in Fig. 8, where we show the field ranges of different vortex states as the ground state of the system. $L \rightarrow L+1$ transition fields obviously increase as $\kappa$ is made smaller, particularly for $\kappa<1$.

In experimental conditions, the applied magnetic field is usually gradually ramped up during the measurement (the so-called sweepup of the magnetic field). Therefore, the

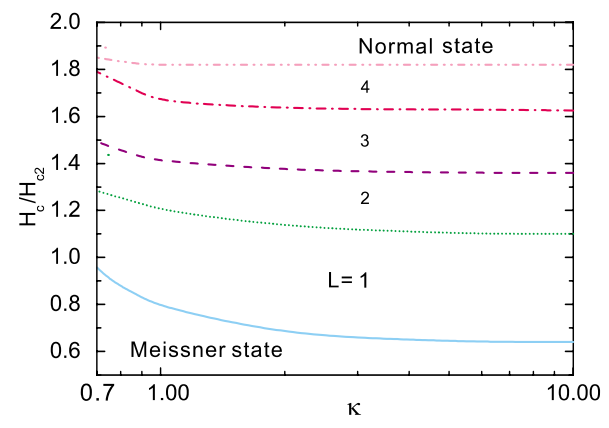

FIG. 8. (Color online) The ground states with different vorticities, in the $H-\kappa$ parameter space, for a sphere with radius $R=3.0 \xi$.

fields at which each vortex penetrates the sample in experiment are different from the ones shown in Fig. 8. Instead, they correspond to the very last points of the stability of each vortex state in the free energy diagram (due to slow field variation and finite Bean-Livingston barrier, transition to the state with lower energy is only possible when the vortex state is no longer stable). Penetration field $H_{p}$ obtained in such a way is plotted as a function of $\kappa$ in Fig. 9. We find similar behavior as in Fig. 8, as $H_{p}$ increases with decreasing $\kappa$. As a main difference, we observed that fourth vortex cannot penetrate the sample for $\kappa<0.83$ when field is swept up, even when the $L=4$ state has lower energy than the state with lower vorticity; instead, we directly find the transition of $L$ $=3$ to the normal state. Still, the $L=4$ state can be recovered in the reversed regime, when magnetic field is swept down, when the maximal number of flux quanta is trapped in the sample after superconductivity nucleates at the equatorial surface of the sample (i.e., the analog of surface superconductivity). Increased $H_{p}$ for all vortex states for lower $\kappa$ follows from the characteristic lengths in the sample. By decreasing $\kappa$, we actually decrease the magnetic penetration length $\lambda$ as the coherence length $\xi$ is kept constant in the simulation. Lower $\lambda$ not only decreases the field penetration in the surface area but also increases the surface energy of the normal domains in the superconductor, i.e., vortices, making their nucleation less energetically favorable.

In order to compare the effect of $\kappa$ on the equilibrium phase diagram, we constructed the $H-R$ phase diagram for $\kappa=1$, as shown in Fig. 10. The first difference to note is that

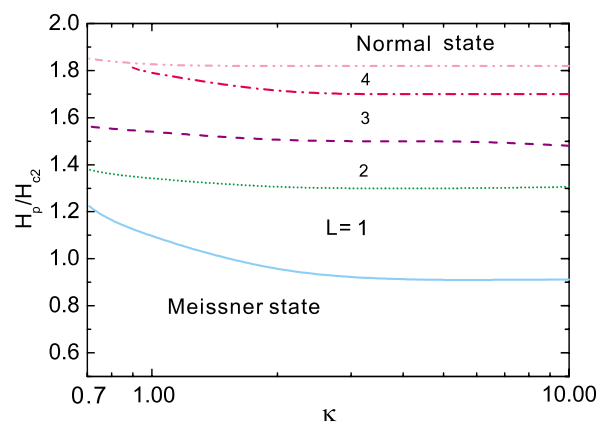

FIG. 9. (Color online) The magnetic fields of successive vortex penetration in the sample of radius $R=3.0 \xi$ as a function of $\kappa$ (for applied magnetic field gradually swept up). 


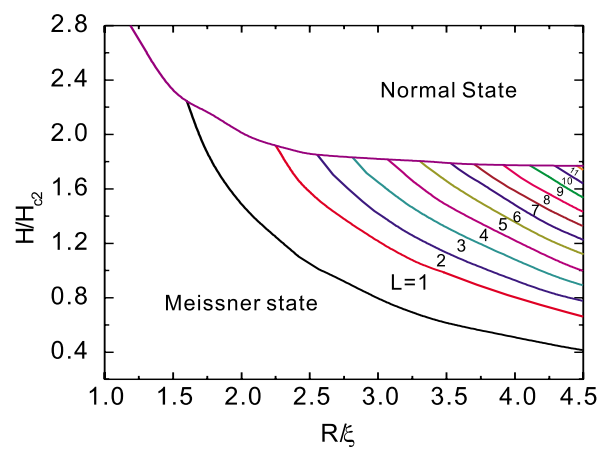

FIG. 10. (Color online) The phase diagram for ground-state vortex configurations as a function of the applied magnetic field $H$ and the radius of the superconducting sphere (for $\kappa=1.0$ ). Areas of stability of different vortex states in the ground state are separated by solid curves.

the maximal number of vortices that can be accommodated by the sample of radius $R$ increases with increasing $\kappa$. For example, in a sphere of radius $R=4.5 \xi, L_{\max }=11$ for $\kappa=1$, while $L_{\max }=12$ for $\kappa=10$. The second important conclusion is that lower $\kappa$ values disfavor multivortex states. In the whole investigated $H-R$ region for $\kappa=1$, only giant-vortex states were found, compared to the rather large multivortex area present in Fig. 5. For clarity, in Fig. 11, we show a direct comparison between two $L=3$ states found in the sphere with $R=4.0 \xi$, but with $\kappa$ equal to 10.0 and 1.0 , respectively, both for applied field $H=0.90 H_{c 2}$. In Fig. 11(a), the phase of the superconducting order parameter changes from 0 to $2 \pi$ in clockwise direction around each of the three identifiable vortex singularities (multivortex), whereas in Fig. 11(b), a triple $0 \rightarrow 2 \pi$ phase change is found around a single singularity (representing a giant vortex).

\section{MAGNETOMETRY RELATED FEATURES}

One of the main properties of the superconductor is its diamagnetism, i.e., the ability to expel magnetic field when cooled below the critical temperature. The degree of field expulsion depends on the sample properties, as well as on the actual superconducting state of the sample. As a measure of the sample's diamagnetism, one can define the resulting magnetic moment or magnetization of the sample. In thermodynamics, magnetization of a sample in magnetic field is defined as the derivative of the Gibbs free energy over the
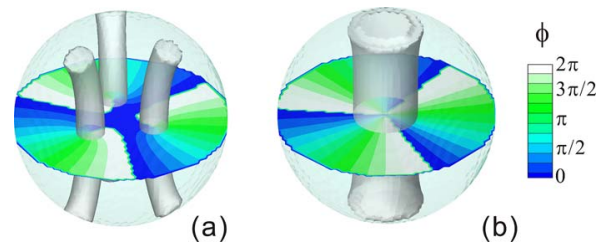

FIG. 11. (Color online) The phase of the order parameter in the equatorial plane (dark/white color- $0 / 2 \pi$ phase) superimposed on the $10 \% 3 \mathrm{D}$ isoplot of the order parameter in the sample for a sphere with radius $R=4 \xi$ at applied field $H=0.90 H_{c 2}$, for (a) $\kappa$ $=10$, and for (b) $\kappa=1$.

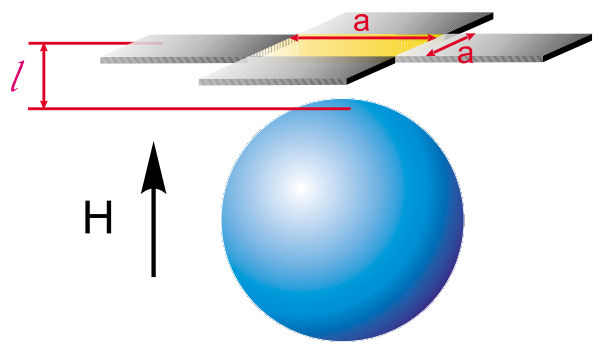

FIG. 12. (Color online) Schematic view of a Hall cross (size of active area $a \times a)$ at distance $l$ above a mesoscopic superconducting sphere with radius $R$.

applied field. ${ }^{32}$ In experiments, on the other hand, magnetization of the sample is measured by magnetometery, and it is taken proportional to the measured voltage on a Hall sensor of particular size. In other words, the measured magnetization equals the amount of flux expelled from the superconductor through a Hall sensor placed at a particular height above the sample. For that reason, in this section, we study the response of such a sensor depending on its size and position, in applied magnetic field $H$, using Eq. (7). For continuity with previous sections, we consider a superconducting sphere with radius $R=4 \xi$, but take for coherence length $\xi$ $=100 \mathrm{~nm}$, and $\kappa=1$ (corresponding to $\mathrm{Nb}$ sample, at $T$ $=0.8 T_{c}$ ). We assume square active area of the Hall cross (see Fig. 12) and consider three different sizes of $160 \times 160$, $320 \times 320$, and $480 \times 480 \mathrm{~nm}^{2}$ and the measurement heights of $40,80,120$, and $160 \mathrm{~nm}$ above the sample. In what follows, we simulate the magnetometry experiments during the sweep up or down of the applied magnetic field.

The magnetization curve following from the thermodynamic definition is plotted in Fig. 13(a), in comparison with the one obtained using Eq. (7). Strong hysteretic behavior is observed due to finite energy barriers for flux entry and/or exit. One can find that the magnetization in Fig. 13(a) is much smaller than that in Figs. 13(b) and 13(c). This is because of the averaging effects of positive and negative local magnetization inside the whole 3D sample.

In Fig. 13(b), we compare the measured signals of the Hall sensors of different sizes. The curves obtained for larger sensors, i.e., $320 \times 320$ and $480 \times 480 \mathrm{~nm}^{2}$ [dashed and dashdotted lines in Fig. 13(b)] behave the same as the magnetization calculated for the sample as a whole in Fig. 13(a) (since the active area of the sensor is almost as large as the cross section of the sample). However, the magnetization curves qualitatively change for the Hall sensor of size 160 $\times 160 \mathrm{~nm}^{2}$ [solid line in Fig. 13(b)]. Namely, on the paths $\mathrm{AE}$ and $\mathrm{CD}$, the magnetization decreases as the applied magnetic field is increased, contrary to the corresponding parts of the other curves in Fig. 13(b). To clearly understand this feature, we investigate the distribution of the superconducting condensate and the magnetic field for particular applied fields, i.e., points $\mathrm{A}-\mathrm{E}$ in Fig. 13(b). Figure 14 shows the magnetic field distribution around the sample [(c) and (d)] (side view and [(e) and (f)] top view) for points $\mathrm{A}$ and $\mathrm{B}$, which reside on the $L=2$ curve [see the phase contourplots in Figs. 14(a) and 14(b)]. The magnetization curve CD corresponds to the $L=4$ vortex state, with properties shown in Fig. 15 . 


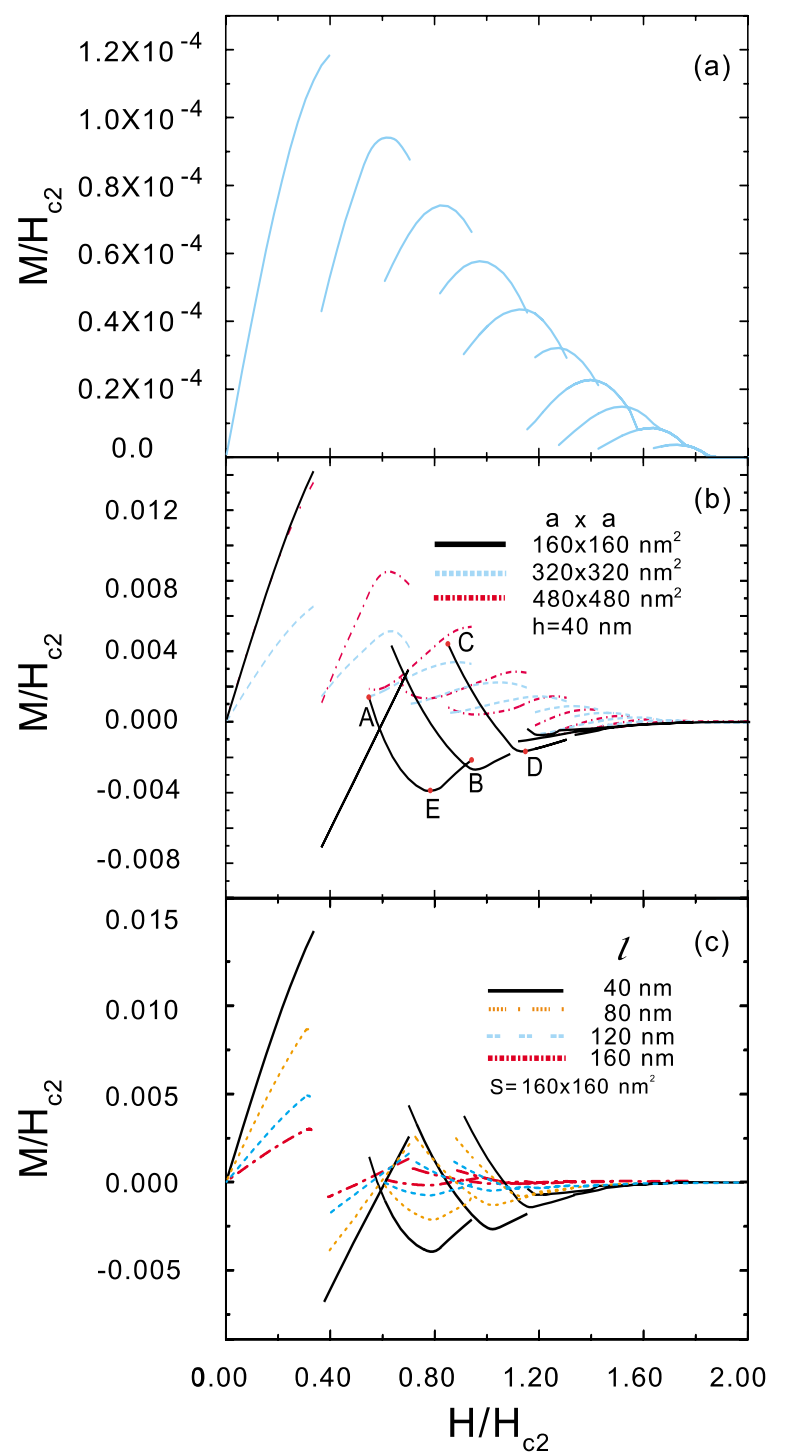

FIG. 13. (Color online) The magnetization curves as a function of the applied magnetic field $H$. (a) The magnetization of the sample as a whole, corresponding the theoretical definition from thermodynamics. (b) Response of the Hall sensors of different size, placed at $40 \mathrm{~nm}$ above the sample. (c) The response of a 160 $\times 160 \mathrm{~nm}^{2}$ probe, when placed at $40,80,120$, and $160 \mathrm{~nm}$ above the sample.

From the phase plots, it is already clear that points B and $\mathrm{D}$ in Fig. 13(b) correspond to the response from the giantvortex states, whereas $\mathrm{A}$ and $\mathrm{C}$ denote the multivortex states. The distributions of the stray magnetic field that the sample generates in response to the applied field at points A and B are plotted in Figs. 14(c)-14(f). Here, dark (light) color indicates positive (negative) peak of the magnetic field.

In the case of the giant vortex [see Figs. 14(d), 14(f), 15(d), and 15(f)], the magnetic field is strongest in the vortex core. With increasing field, the screening currents flowing along the sample edges increase and compress the vortex in the center. Therefore, with increasing field, the Hall sensor sitting right above the vortex detects more of the screening field of the Meissner currents, and the measured magnetiza-

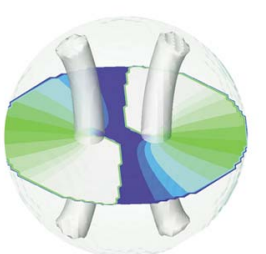

(a)

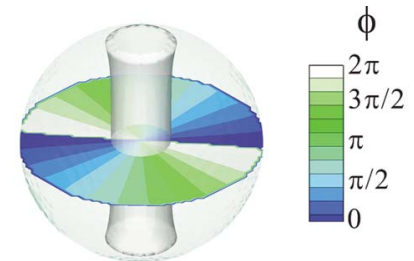

(b)

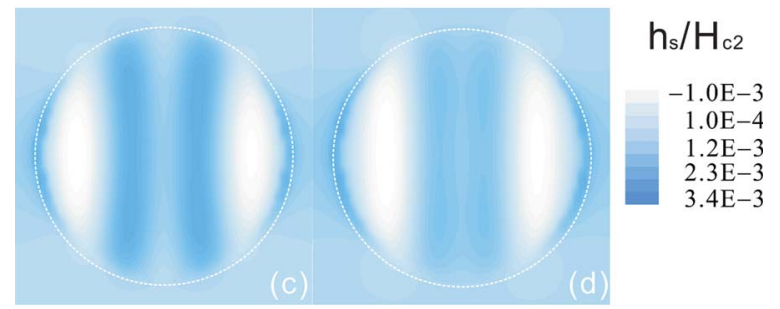

(e)

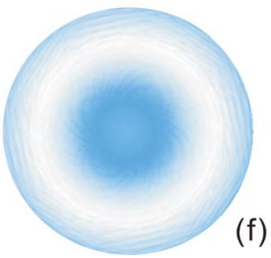

FIG. 14. (Color online) [(a) and (b)]. The phase in the equatorial plane and 3D isoplot of the order parameter in the sample for points (a) A and (b) B in Fig. 13(b) (showing the MV and GV states for $L=2)$. [(c)-(e)] Corresponding field distribution around the superconducting sphere for points [(c) and (e)] A and [(d) and (f)] B. Side view of the sample is shown in (c) and (d), and top view in (e) and (f). In all graphs, only the response field of the superconductor is plotted $\left(\overrightarrow{h_{s}}=\vec{h}-\vec{H}\right)$, changing from negative (light color) to a positive extreme (dark color). Sample edge is indicated by the white dashed line.

tion increases. On the other hand, in the case of the multivortex [see Figs. 14(c), 14(e), 15(c), and 15(e)], the stray magnetic field has a local minimum in the center of the sample, between the vortices. In a ring distribution of the vortices (each with a vortex current circulating around it), the current in the central region effectively has the antivortex direction, thus the same as the screening current at the edge. With increasing applied magnetic field, vortices are compressed closer together, which now decreases the magnetization signal coming from the central antivortexlike currents. Therefore, if we look back at the AB curve in Fig. 13(b), we can conclude that along the $\mathrm{AE}$ path, we have a multivortex state, whereas along the EB path, a giant-vortex state is formed. For the same reason, the curve found for $L=4$ state demonstrates a multivortex state on the shown CD path. To conclude, the concave shape of the magnetization curves measured by a comparatively small Hall sensor indicates the multi- to giant-vortex transition at the inflexion point of the curve.

That the shown distinction between the giant- and multivortex states in the magnetization curves is not an accidental property we illustrate further in Fig. 13(c). There, we show the magnetization curves measured by the $160 \times 160 \mathrm{~nm}$ Hall sensor placed at different heights above the sample. Of course, closer proximity of the sensor to the sample is always 

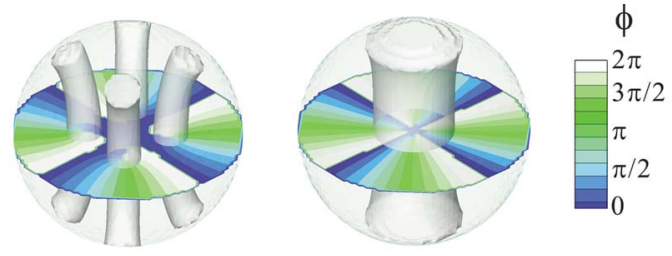

(a)

(b)

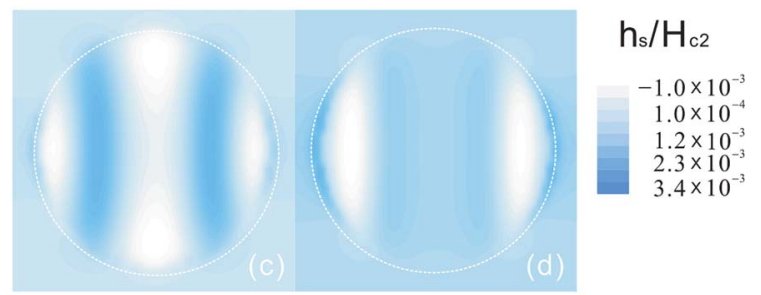

(e)

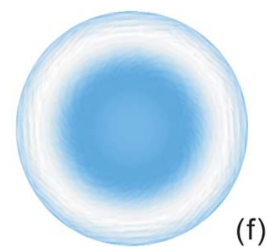

FIG. 15. (Color online) Same as in Fig. 14, but correspond to points (a) C and (b) D in Fig. 13(b).

desired, as the signal is more pronounced. However, Fig. 13(c) clearly shows that the features of the magnetization curves are maintained even for larger separation between the Hall bar and the superconducting sample. At higher measurement heights, GV-MV distinction is still feasible as long as the signal does not succumb below the noise level.

\section{CONCLUSIONS}

In this work, superconducting and magnetic properties of mesoscopic superconducting spheres are studied. Full three dimensionality of the problem is taken into account, and the two coupled nonlinear Ginzburg-Landau equations are solved self-consistently on a 3D grid, with demagnetization effects being fully considered. Found vortex states illuminate the $3 \mathrm{D}$ aspect of the study; although they form structures essentially similar to the ones found earlier in superconducting disks, vortices tend to bend in the equatorial plane of the sphere while remaining perpendicular to the spherical surface of the sample at the points of entry and exit. Vortex configurations are studied in detail for different radii of the sample and the equilibrium vortex phase diagram is shown for a type-II sample. Both giant- and multivortex states are found. However, multivortex states are found unstable with respect to lower values of the Ginzburg-Landau parameter $\kappa$. In other words, the appearance of multivortex states is not determined only by the sample size (i.e., confinement) but also by the choice of the superconducting material. The latter also influences the magnetic response of the sample, which we studied considering the known experimental techniques. In a hypothetic experiment, we calculated the response of a Hall bar with square active area as a function of its size and the measurement height above the sample. We found distinct features of the magnetization curves measured by comparatively small Hall probes, which can be used to experimentally distinguish the giant-vortex from the multivortex states.

\section{ACKNOWLEDGMENTS}

This work was supported by the Flemish Science Foundation (FWO-Vl), the Interuniversity Attraction Poles (IAP) Programme-Belgian State-Belgian Science Policy, ESF-JSPS NES program, and the ESF-AQDJJ network. M.V.M. acknowledges support from the EU Marie Curie IntraEuropean program.

*francois.peeters@ua.ac.be

${ }^{1}$ H. Kupfer, G. Linker, G. Ravikumar, T. Wolf, A. Will, A. A. Zhukov, R. Meier-Hirmer, B. Obst, and H. Wuhl, Phys. Rev. B 67, 064507 (2003).

${ }^{2}$ E. T. Filby, A. A. Zhukov, P. A. J. de Groot, M. A. Ghanem, P. N. Bartlett, and V. V. Metlushko, Appl. Phys. Lett. 89, 092503 (2006).

${ }^{3}$ Z.-L. Xiao, C. Han, W.-K. Kwok, H.-H. Wang, U. Welp, J. Wang, and G. Crabtree, J. Am. Chem. Soc. 126, 2316 (2004).

${ }^{4}$ V. A. Schweigert and F. M. Peeters, Phys. Rev. B 57, 13817 (1998).

${ }^{5}$ V. A. Schweigert, F. M. Peeters, and P. S. Deo, Phys. Rev. Lett. 81, 2783 (1998).

${ }^{6}$ J. J. Palacios, Phys. Rev. B 58, R5948 (1998).

${ }^{7}$ J. J. Palacios, Phys. Rev. Lett. 84, 1796 (2000).

${ }^{8}$ A. I. Buzdin and J. P. Brision, Phys. Lett. A 196, 267 (1994).

${ }^{9}$ V. V. Moshchalkov, L. Gielen, C. Strunk, R. Jonckheere, X. Qiu, C. V. Haesendonck, and Y. Bruynseraede, Nature (London) 373, 319 (1995).

${ }^{10}$ V. Bruyndoncx, L. Van Look, M. Verschuere, and V. V. Mosh-

chalkov, Phys. Rev. B 60, 10468 (1999).

${ }^{11}$ A. K. Geim, I. V. Grigorieva, S. V. Dubonos, J. G. S. Lok, J. C. Maan, A. E. Filippov, and F. M. Peeters, Nature (London) 390, 259 (1997)

${ }^{12}$ A. K. Geim, S. V. Dubonos, I. V. Grigorieva, K. S. Novoselov, F. M. Peeters, and V. A. Schweigert, Nature (London) 407, 55 (2000).

${ }^{13}$ A. Kanda, B. J. Baelus, F. M. Peeters, K. Kadowaki, and Y. Ootuka, Phys. Rev. Lett. 93, 257002 (2004).

${ }^{14}$ B. J. Baelus, A. Kanda, F. M. Peeters, Y. Ootuka, and K. Kadowaki, Phys. Rev. B 71, 140502(R) (2005).

${ }^{15}$ J. Bonca and V. V. Kabanov, Phys. Rev. B 65, 012509 (2001).

${ }^{16}$ B. J. Baelus and F. M. Peeters, Phys. Rev. B 65, 104515 (2002).

${ }^{17}$ A. S. Mel'nikov, I. M. Nefedov, D. A. Ryzhov, I. A. Shereshevskii, V. M. Vinokur, and P. P. Vysheslavtsev, Phys. Rev. B 65, 140503(R) (2002).

${ }^{18}$ V. R. Misko, V. M. Fomin, J. T. Devreese, and V. V. Moshchalkov, Phys. Rev. Lett. 90, 147003 (2003).

${ }^{19}$ M. Morelle, G. Teniers, L. F. Chibotaru, A. Ceulemans, and V. V. Moshchalkov, Physica C 369, 351 (2002). 
${ }^{20}$ M. Morelle, J. Bekaert, and V. V. Moshchalkov, Phys. Rev. B 70, 094503 (2004).

${ }^{21}$ B. J. Baelus, A. Kanda, N. Shimizu, K. Tadano, Y. Ootuka, K. Kadowaki, and F. M. Peeters, Phys. Rev. B 73, 024514 (2006).

${ }^{22}$ A. K. Elmurodov, D. Y. Vodolazov, and F. M. Peeters, Europhys. Lett. 74, 151 (2006).

${ }^{23}$ F. Mohamed, M. Troyer, G. Blatter, and I. Luk'yanchuk, Phys. Rev. B 65, 224504 (2002).

${ }^{24}$ B. J. Baelus, D. Sun, and F. M. Peeters, Phys. Rev. B 75, 174523 (2007).

${ }^{25}$ Q. Du and L. Ju, J. Comput. Phys. 201, 511 (2004).

${ }^{26}$ M. M. Doria, A. R. de C. Romaguera, and F. M. Peeters, Phys. Rev. B 75, 064505 (2007).
${ }^{27}$ A. de C. Romaguera, M. M. Doria, and F. M. Peeters, Phys. Rev. B 76, 020505(R) (2007).

${ }^{28}$ R. Kato, Y. Enomoto, and S. Maekawa, Phys. Rev. B 47, 8016 (1993).

${ }^{29}$ U. Schumann and R. A. Sweet, J. Comput. Phys. 75, 123 (1988).

${ }^{30}$ B. J. Baelus, F. M. Peeters, and V. A. Schweigert, Phys. Rev. B 63, 144517 (2001).

${ }^{31}$ N. E. Hussey, J. R. Cooper, R. A. Doyle, C. T. Lin, W. Y. Liang, D. C. Sinclair, G. Balakrishnan, D. M. Paul, and A. Revcolevschi, Phys. Rev. B 53, 6752 (1996).

${ }^{32}$ P. G. de Gennes, Superconductivity of Metals and Alloys (Addison-Wesley, Reading, MA, 1989). 